\title{
Image Fusion Based on Wavelet Transforms
}

\author{
Laxman Tawade $^{1}$, Abida Bapu Aboobacker ${ }^{2}$ and Firdos Ghante ${ }^{1}$ \\ ${ }^{1}$ VDF School of Engineering and Technoloy, Latur India \\ ${ }^{2}$ Calicut University, India \\ tawadelaxman561988@gmail.com,abbibappu@gmail.com
}

\begin{abstract}
The image fusion is the process of combining relevant information from two or more images into a single image. The resulting image will be more informative than any of the input images. A new approach for object extraction from high-resolution images is presented in this report. In this paper, we have presented image fusion based on wavelet transform.
\end{abstract}

Keywords: Image Fusion, discrete wavelet transforms (DWT)

\section{Introduction}

Medical Image Fusion is a sort of data fusion and developed from that into a new data fusion technology. As an advanced method of image processing technology to integrate multi-source image data, image fusion is to integrate two or more images into a new fusion image. The aim of fusion is the combination of images to integrate the information of each individual technique and reduce the uncertainty of the image information. Computed tomography (CT), and positron emission tomography (PET) provide data conditioned by the different technical, anatomical and functional properties of the organ or tissue being studied, with values of sensitivity, specificity and diagnostic accuracy variations between them. Their fusion enables the "unification" of the various technique-dependent data, thus "summing" the diagnostic potential of each individual technique. Because of the image fusion technology which can effectively integrate the image information, the fusion images are more intelligible and readable and have more information than the images that are got through single channel, and this technology has been concentrated very much, and has had a great development.

Truly the image fusion is the process of combining relevant information from two or more images into a single image. The resulting image will be more informative than any of the input images. A new approach for object extraction from high-resolution images is presented in this report. In this we present a new approach to better extract information from CT (Computed Tomography) /PET (positron emission tomography) which will be helpful to diagnose diseases. Since there are various methods and algorithms for fusion of these images and as there are special advantages for each algorithm along with ever growing need to use of this technique, as a result research about this field of study becomes more sophisticated. Many methods exist to perform image fusion. The very basic ones are the Probabilistic Approximation model. The wavelet approach gives better accuracy and increased information compared to the previous techniques.

\section{CT/PET Image fusion:}

Above methods are used for merging CT/PET images. In this we can have two types of images, 


\section{1) Computed Tomography (CT) image :}

CT image supports clear bones information but no soft tissues information.

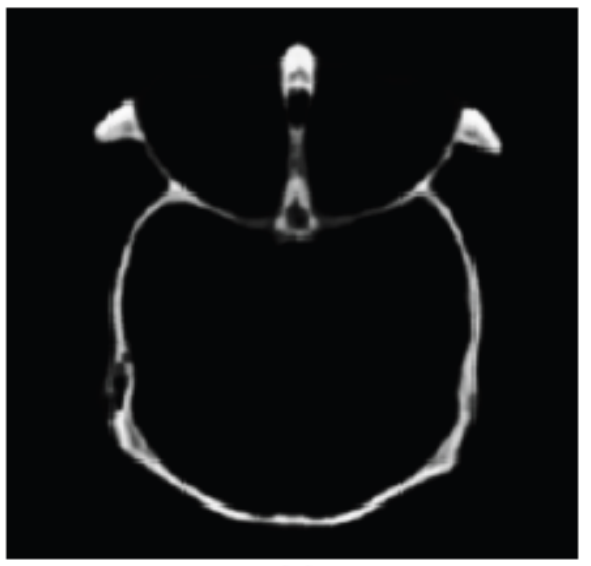

Figure 1.1. Original CT Image

\section{What is it?}

A computed tomography (CT) scan, also called a CAT scan, uses x-rays to take pictures of the head from many different angles. The pictures provide a detailed, cross-sectional view of specific areas of the brain. CT scans are widely used and less expensive than other scanning technologies. A CT scan shows changes in bone better than any other imaging method. It is also the only scanning technique that shows images of bone, blood vessels etc. at the same time. A CT scan is often used in emergency rooms because it can be performed quickly to screen people who have had some type of trauma, a stroke or other life-threatening condition.

\section{Why is it performed?}

CT scans are used to help diagnose many medical conditions including strokes, head trauma, tumors, hydrocephalus, blood clots, cerebral atrophy, internal bleeding, skull fractures, brain aneurysms, and hearing loss. CT scans are not used to diagnose Alzheimer's disease. They are used to confirm or rule out other causes of dementia in people who show signs of memory loss.

\section{2) Positron Emission Tomography (PET) image:}

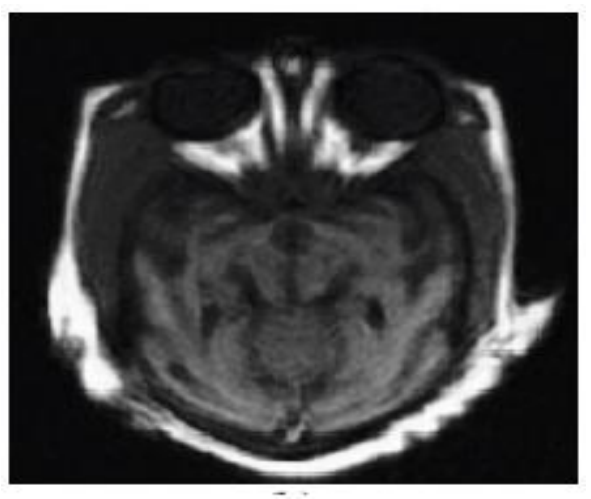

Figure 1.2. Original PET Image 
A PET image helps to diagnose various diseases such as brain tumors or torn ligaments. PET scan is a test that provides very clear pictures of structures inside the body. PET image provides clear soft tissues information but no bones information. That is to say, the same object in the two medical images appears very distinctly. Hence, in order to support more accurate information for diagnosis and treatment it is necessary to fuse them by using their complementary information. According to this analysis of the CT and PET images, we performed the wavelet coefficients of the low frequency band and high frequency bands with different fusion rules separately. Another important factor we must consider is that in fact each pixel in the image has some relation with its neighboring pixels, which means every pixel is affected by its neighboring pixels. Therefore, for selecting the slice in focus at coordinate $(x, y)$, a neighborhood of each pixel should be also taken into account.

Here we consider only two source images, $X$ and $Y$, and the fused image $Z$. The method can be easily extended to more than two images. Generally, an image $I$ have its multiscale decomposition (MSD) representation denoted as Diand the activity level as $A i$. Hence we will encounter $D x, D y, D z, A x$, and $A y$. Let $p=(m, n, k, l)$ indicate the index corresponding to a particular MSD coefficient, where $m$ and $n$ indicate the spatial position in a given frequency band, $k$ is the decomposition level and $l$ is the frequency band of the MSD representation. Thus, $D l(p)$ and $A l(p)$ are the MSD value and activity level of the corresponding coefficient, respectively.

\subsection{Literature survey:}

Image fusion as a kind of information-integrated technology has been used in many fields recently. For medical images, the medical machine of PET/CT makes the within-machine fusion come true. Although the fusion image not only has the character of CT that can make a precise location of pathological changes but also has the character of PET that can detect the pathological as early as possible. As the within-machine fusion equipment's are very expensive, it leads a high fee. Only some large hospitals are equipped with the withinmachine fusion equipment. So a large space has been left to the development of fusion differences. Based on the wavelet-transform, the fusion of the CT image and PET image comes true. In the end, we gain the fusion CT/PET image. We can detect and locate the pathological changes exactly and immediately in the fusion image. And actually, this is the predominance of the fusion image. Today various countries use image fusion technology in a widespread way to collect medical data to study and diagnose diseases. It is necessary to combine the data in order to use the existing and available data optimally. While preserving the spectral specifications the combined new data enjoy a high degree of spatial resolution. In medical applications, the increasing use of CT/PET gives a motivation for different image fusion algorithms.

Several situations in image processing require high spatial and high spectral resolution in a single image. Most of the available equipment is not capable of providing such data convincingly. The image fusion techniques allow the integration of different information sources. The fused image can have complementary spatial and spectral resolution characteristics. But, the standard image fusion techniques can distort the spectral information of the multispectral data, while merging. Several situations in image processing simultaneously require high spatial and high spectral information in a single image. This is important in medical applications for diagnose diseases. 


\subsubsection{Present Scenario:}

Some other well-known image fusion methods are:

- High pass filtering technique

- IHS transform based image fusion

- PCA based image fusion

- Pair-wise spatial frequency matching

\subsubsection{Limitations of above system:}

The major drawback of all the methods described is the need for an additional more highly resolved band. In fact it is possible to gain the essential spatial resolution data by employing a sub-pixel displacement of the simultaneously acquired spectral bands.

\section{System Development}

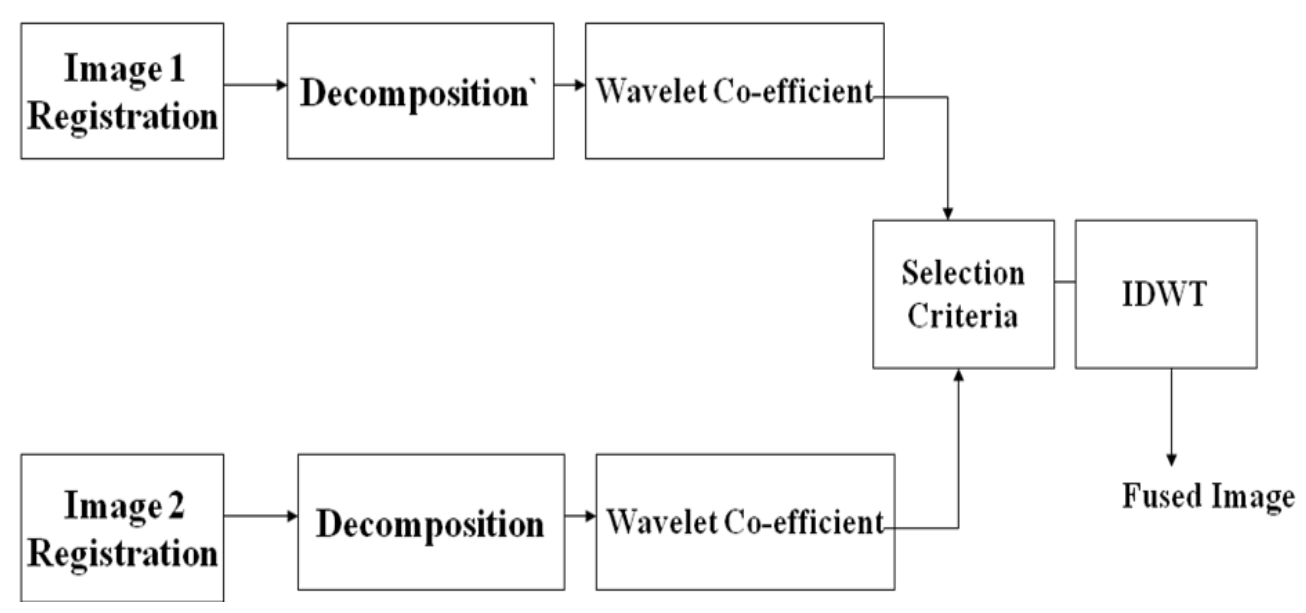

Figure 2.1. Block Diagram of Image Fusion

\subsection{Block Diagram Description:}

\subsubsection{Image Registration:}

One of main issue in image fusion of CT/MRI images is image alignment, which refers to pixel-by-pixel alignment of images. Image is nothing but matrix. For the resultant output image to be distinct and have a better visualization, both images should have same size. Same size of images also becomes a basic requirement for point to point correspondence between two pixel values. When images are co-registered, you can think of overlaying one on top of the other and examining any pixel in the top of image.

\subsubsection{Decomposition by DWT:}

\section{Why wavelet?}

Often times, the information that cannot be readily seen in the time domain can be seen in the frequency domain. Today Fourier transforms are used in many different areas including all branches of engineering. Although FT is probably the most popular transform being used, it is not the only one. There are many other transforms that are used quite often by engineers 
and mathematicians. Hilbert transform, STFT, Wigner distributions, Radon transforms and off-course featured transformation, the Wavelet transform, constitute only a small portion of a huge list of transform that are available at Engineer's disposal.

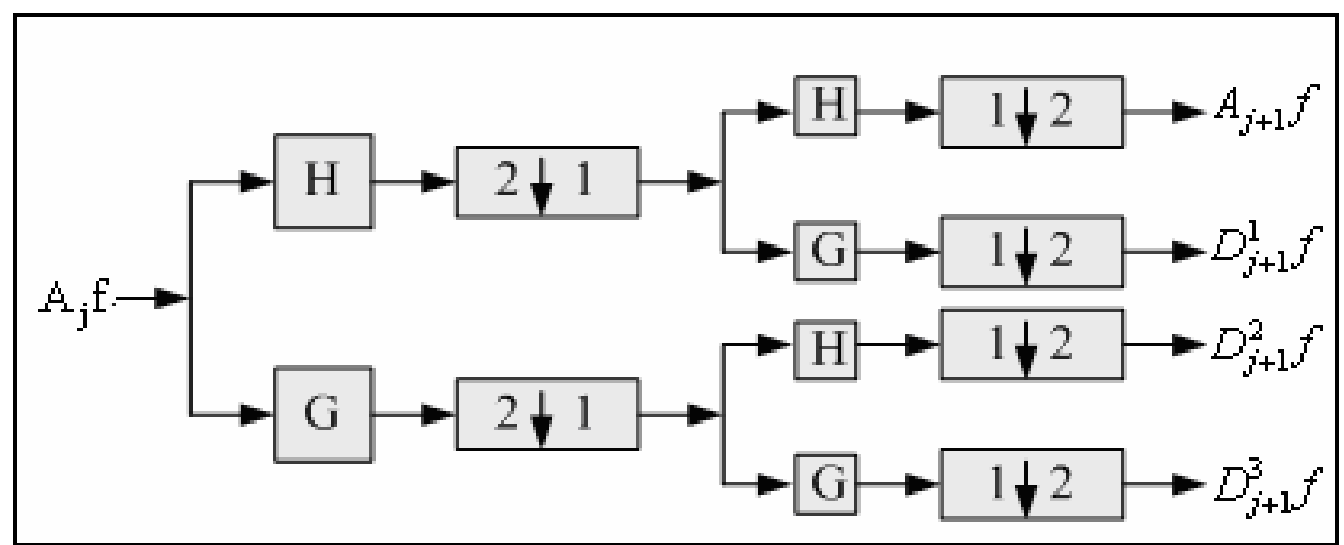

Figure 2.2. Image Decomposition Algorithm using 2-D Discrete Wavelet Transform

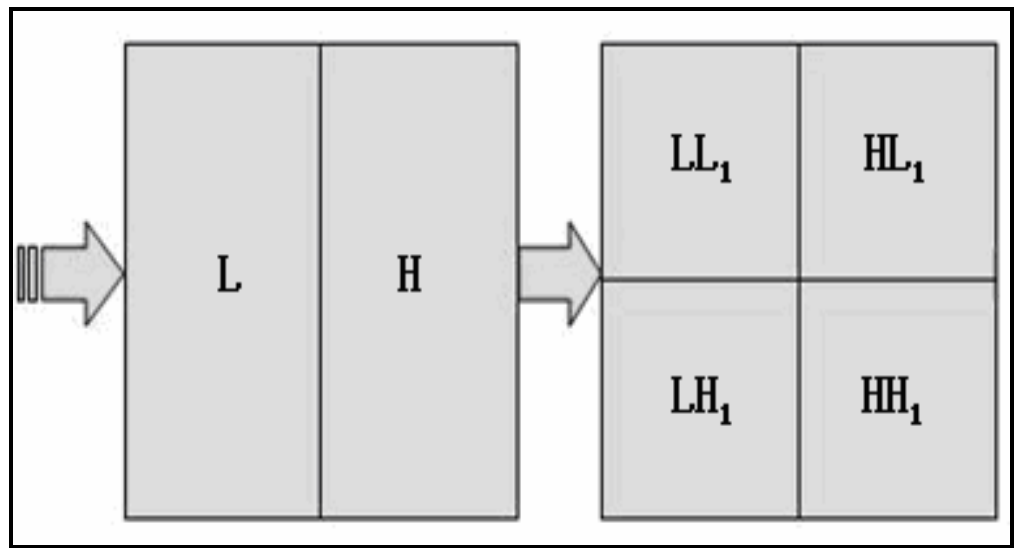

Figure 2.3. Image Decomposition Schematic Diagram using 2-D Discrete Wavelet Transform

\subsubsection{Low Frequency Band Fusion:}

Since the low frequency band is the original image at coarser resolution level, it can be considered as a smoothed and subsampled version of the original image. Based on the pervious analysis of the characteristics of the CT \& MR images, here for the low frequency band, a maximum-selection (MS) fusion rule to produce a single set of coefficients is used firstly. The scheme selects the largest absolute wavelet coefficient at each location from the input images as the coefficient at that location in the fused image:

$$
\begin{aligned}
& A_{I}(p)=\left|D_{I}(p)\right| \\
& A_{i}(p)=\max \left(A_{X}(p), A_{Y}(p)\right)
\end{aligned}
$$


After the MS selection, we will compute neighboring coefficients in the composite MSD by applying consistency verification. In our own research, a window-based verification (WBV) is performed. The WBV employs a small window (here a $3 \times 3$ window) centered at the current coefficient position. The WBV rule is that if the center composite MSD coefficient comes from image $X$, but the majority of the surrounding coefficients in the window come from image $Y$, then the center sample and its neighboring samples are changed to come from $Y$.

\subsubsection{High Frequency Bands Fusion:}

For the high frequency bands, since the purpose of image fusion requires that the fused image must not discard any useful information contained in the source images and effectively preserve the details of input images such as edges and textures. It is generally believed that the details of an image are mainly included in the high frequency of the image. Therefore, it is important to find appropriate methods to merge the details of input images. The conventional selection of high frequency coefficients only depend on their absolute value without taking any consideration of the neighboring coefficients. However, as we know a pixel in the image must have some relation with its neighboring pixels, which can reveal a MSD coefficient also has relations with its neighboring MSD coefficients. Based on this analysis, we use a maximal local variance scheme to select the high frequency coefficients:

$$
\begin{gathered}
m_{i}(x, y)=\frac{1}{M \times N} \sum_{s=-N / 2}^{N / 2} \sum_{t=-M / 2}^{M / 2} D_{i}(x+s, y+t) \\
\sigma_{i}(x, y)=\frac{1}{M \times N} \sum_{s=-N / 2}^{N / 2} \sum_{t=-M / 2}^{M / 2}\left(\begin{array}{l}
D_{i}(x+s, y+t) \\
-m_{i}(x, y)
\end{array}\right)^{2} \\
A_{i}(p)= \begin{cases}D_{X}(p) & \sigma_{X}(x, y) \geq \sigma_{Y}(x, y) \\
D_{Y}(p) & \sigma_{X}(x, y)<\sigma_{Y}(x, y)\end{cases}
\end{gathered}
$$

Where $M \times N$ is the neighboring size and here it is set as $3 \times 3, m(x y) i,(x y) i \sigma$, denote the mean value and variance value of the coefficients in the window of $M \times N$ respectively. We must pay attention to that the high frequency bands referred to include the vertical, horizontal, and diagonal high frequencies of the image, respectively. Therefore, the fusion process should be performed in all these domains. The procedures of our method can be summarized as the following.Register the CT and PET images decompose the images to 3-4 wavelet planes (resolution levels).The wavelet coefficients of the low frequency are performed by followed by the above consistency verification rule. The wavelet coefficients of the high frequency are

selected by equations. Perform the inverse wavelet transform with the combined coefficients obtained.

\subsubsection{Fusion rules:}

Three previously developed fusion rule schemes were implemented using discrete wavelet transform based image fusion:

\subsubsection{Maximum Selection (MS) Scheme:}

This simple scheme just picks the coefficient in each subband with the largest magnitude. 


\subsubsection{Weighted Average (WA) Scheme:}

This scheme developed by Burt and Kolczynski uses a normalized correlation between the two images' sub bands over a small local area. The resultant coefficient for reconstruction is calculated from this measure via a weighted average of the two images' coefficients.

\subsubsection{Window Based Verification (WBV) Scheme:}

This scheme developed by Li et al. creates a binary decision map to choose between each pair of coefficients using a majority filter.

\subsubsection{Extraction and Concatenation of Coefficients:}

After applying specified level of decomposition using DWT, the approximate and detail coefficients are extracted. The detail coefficients are further filtered to increase luminance. After filtering of coefficients, the approximate coefficient of CT/PET images is concatenated. This is the step where the actual image fusion takes place.

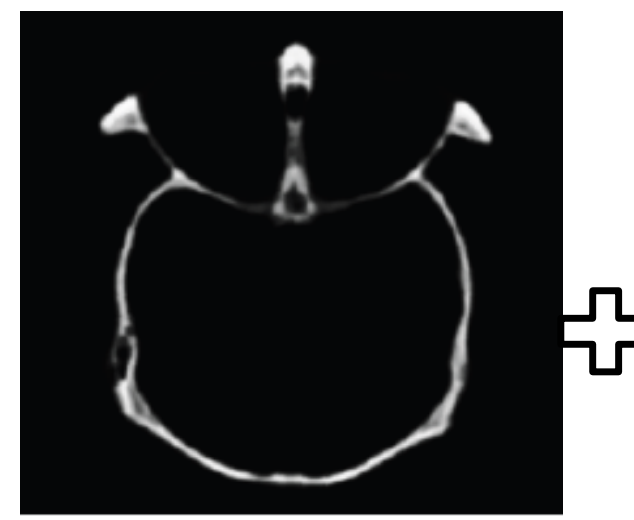

CT Image
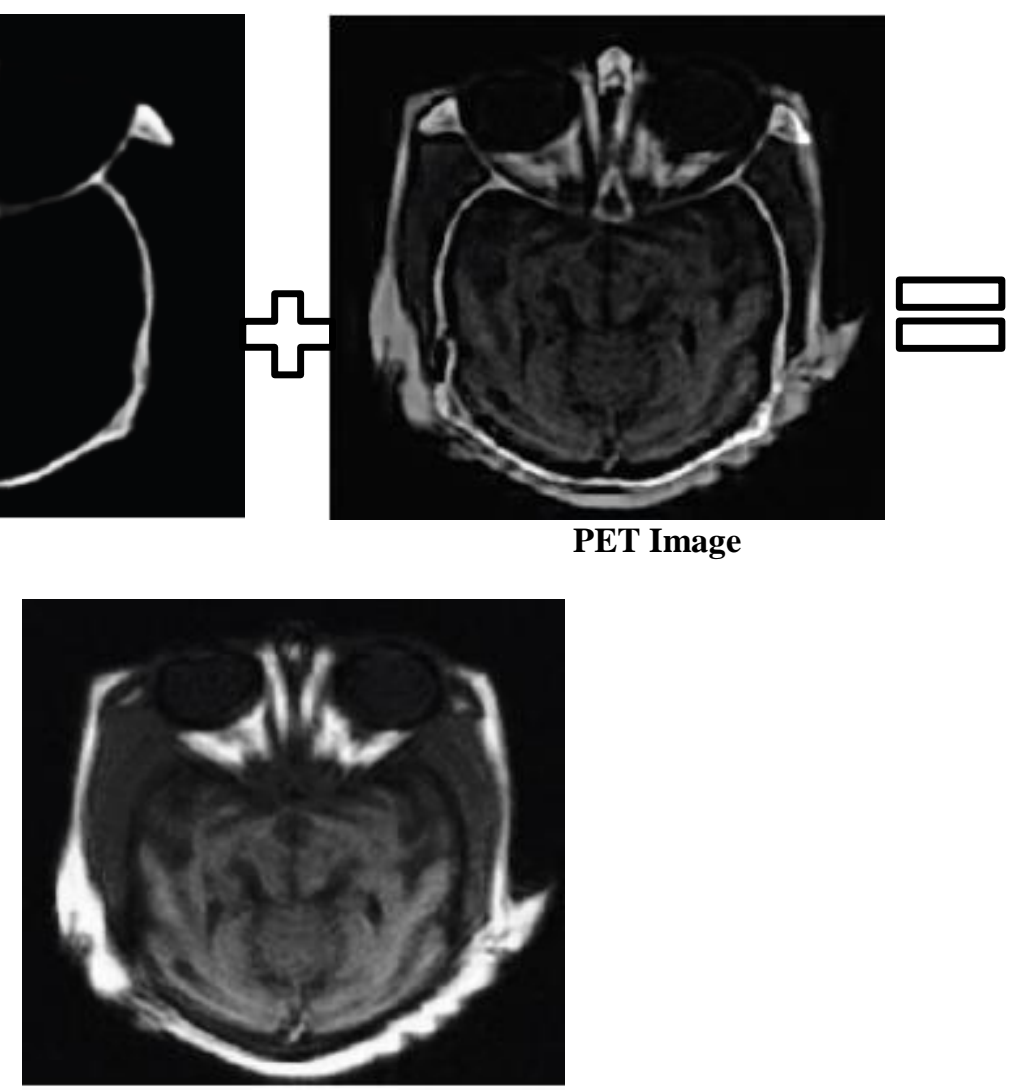

FUSED Image

Figure 2.4. Image Fusion

\subsubsection{Reconstruction using IDWT:}

After concatenation, the inverse discrete wavelet transform is applied to get resultant output image after fusion. 


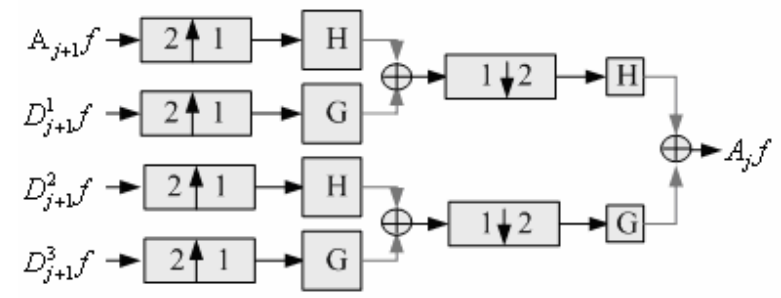

Figure 2.5. Reconstruction using IDWT

\section{Algorithms}

\subsection{Algorithm for multilevel decomposition:}

1. Take the CT and PET images from user.

2. Check for the size.

3. If size is not same, resize the input images.

4. Enter the level of decomposition.

5. Decompose both the images by using discrete wavelet transform.

6. Separate out the approximate and details from decomposed image.

7. Apply appropriate selection criterion to select the wavelet coefficients from both the images and concatenate them to for a single decomposed image.

8. Reconstruct the image by using inverse discrete wavelet transform.

9. Evaluate the different parameters.

10. Display the input and output images.

\subsection{Algorithm for absolute coefficient decomposition:}

1. Take the CT and PET images from user.

2. Check for the size.

3. If size is not same, resize the input images.

4. Enter the level of decomposition.

5. Enter the type of wavelet (haar, daubechies, symlet).

6. Decompose both the images by using discrete wavelet transform.

7. Separate out the approximate and details from decomposed image.

8. Apply appropriate selection criterion to select the wavelet coefficients from both the images and concatenate them to form a single decomposed image.

9. Take the absolute values of approximate and details.

10. Reconstruct the image by using inverse discrete wavelet transform.

11. Evaluate the different parameters.

12. Display the input and output images.

\subsection{Algorithm for Gradient Coefficient Decomposition:}

1. Take the CT and PET images from user.

2. Check for the size.

3. If size is not same, resize the input images.

4. Enter the level of decomposition.

5. Enter the type of wavelet (haar, daubechies, symlet).

6. Decompose both the images by using discrete wavelet transform. 
7. Separate out the approximate and details from decomposed image.

8. Apply appropriate selection criterion to select the wavelet coefficients from both the images and concatenate them to form a single decomposed image.

9. Take the gradient values of approximate and details.

10. Reconstruct the image by using inverse discrete wavelet transform.

11. Evaluate the different parameters.

12. Display the input and output images.

\subsection{Algorithm for removal of negative coefficient decomposition:}

1. Take the CT and PET images from user.

2. Check for the size.

3. If size is not same, resize the input images.

4. Enter the level of decomposition.

5. Enter the type of wavelet (haar, daubechies, symlet).

6. Decompose both the images by using discrete wavelet transform.

7. Separate out the approximate and details from decomposed image.

8. Apply appropriate selection criterion to select the wavelet coefficients from both the images and concatenate them to form a single decomposed image.

9. Set the negative approximate and details to zero.

10. Reconstruct the image by using inverse discrete wavelet transform.

11. Evaluate the different parameters.

12. Display the input and output images.

\subsection{Flow Chart:}

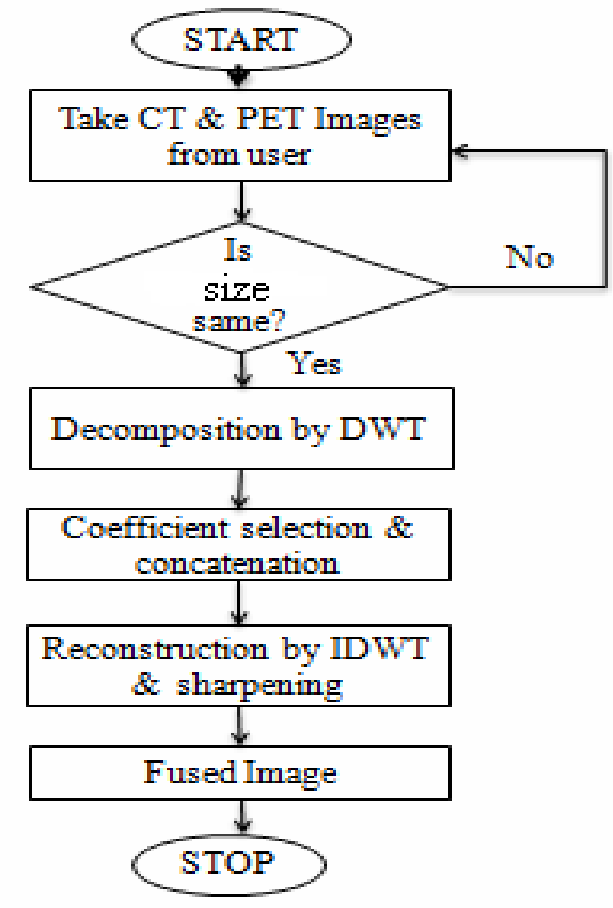

Figure 3.1. Flow chart of Algorithm 


\subsection{Implementation Steps in Matlab:}

1. Select 1st input image (CT image) from the location which is of size $m^{*} n$.

2. Select $2^{\text {nd }}$ input image (PET image) from the location which is of same size $m * n$.

3. Decompose both the images by using discrete wavelet transform.

4. Select the wavelet coefficients from both the images and concatenate them to form a single decomposed image.

5. Reconstruct the image by using inverse discrete wavelet transform.

6. Use sharpening algorithm to get the final fused image.

\subsection{Implementation steps in $\mathrm{VC}++$ :}

1. Create empty project

2. Add library files of CV through linker.

3. Add source files.

4. Add header files.

5. Add images read to be computed.

6. In the main.cpp, write program.

7. Main function: write all declarations.

8. Call the subfunctions of decompose and reconstruct.

\section{Results and Conclusion}

\subsection{Result:}

The results obtained after $3^{\text {rd }}$ level decomposition of input images for following wavelet types:

Haar: (haar)

CT

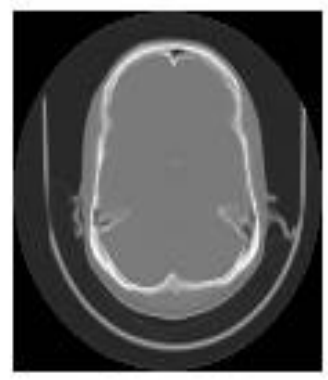

PET

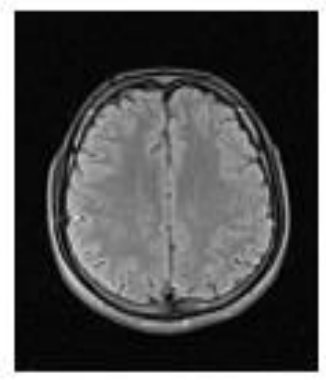

\section{Fused}

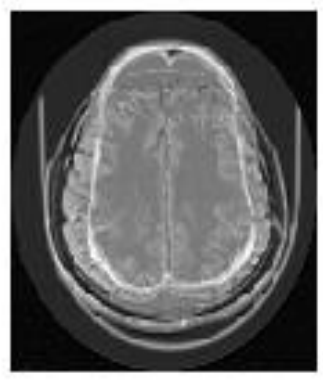

Figure 4.1. Fusion of Images by Haar 

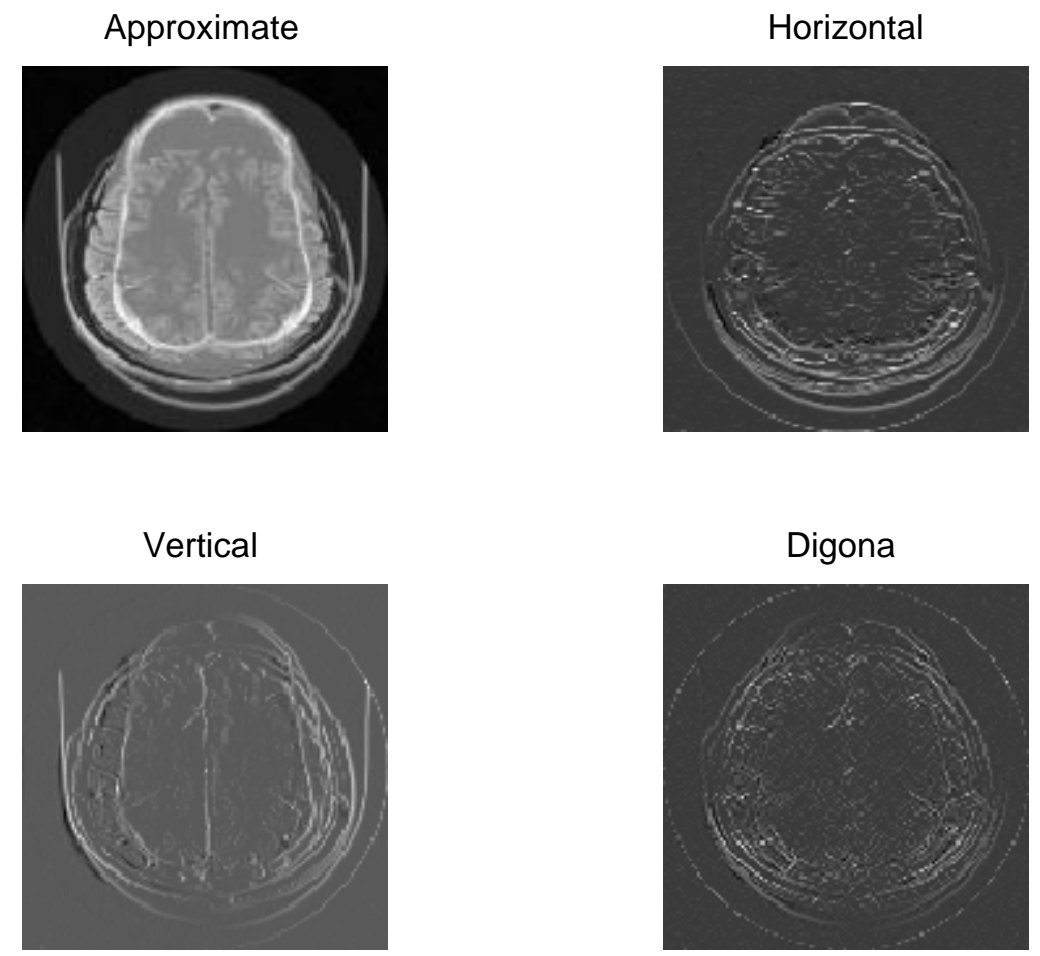

Figure 4.2. Decomposition of Images by haar

Daubecies: (db3)
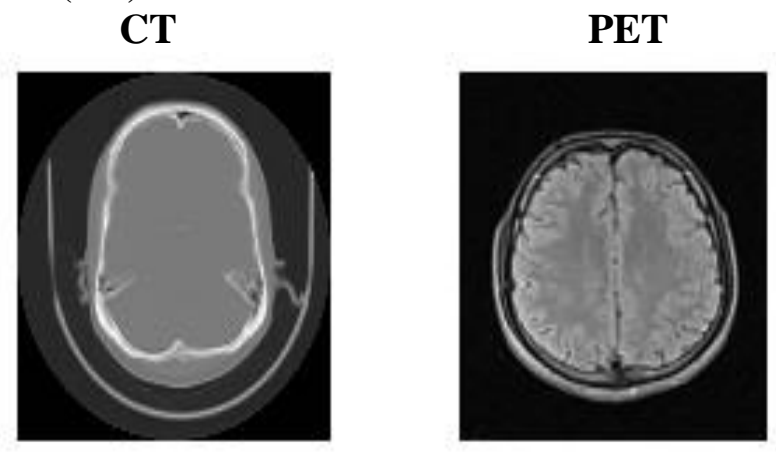

Fused

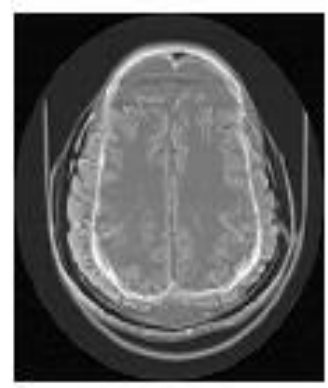

Figure 4.3. Fusion of Images by Daubechies 

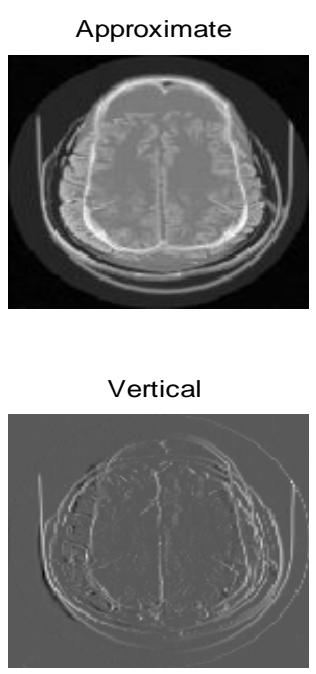

Horizontal

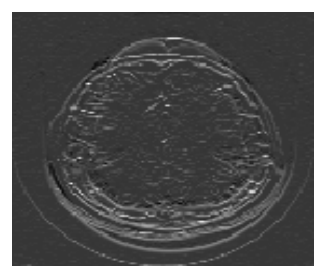

Digonal

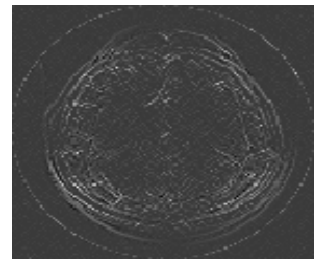

Figure 4.4. Decomposition of Images by Daubechies

Symlet: (sym7)
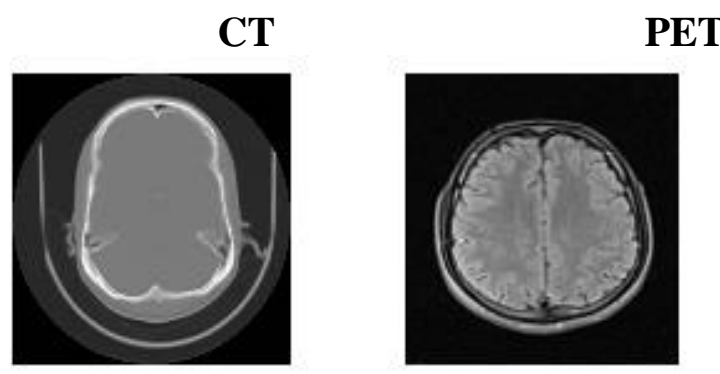

Fused

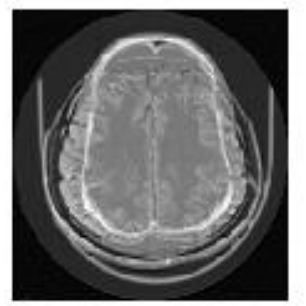

Figure 4.5. Fusion of Images by Symlet

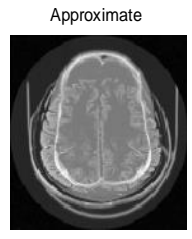

Vertical

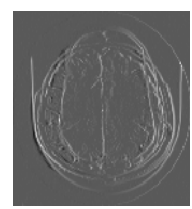

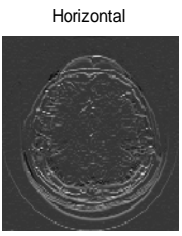

Digonal

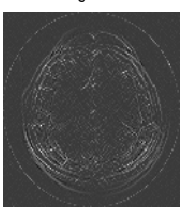

Figure 4.6. Decomposition of Images by Symlet 


\subsection{Parameter Evaluation:}

The different parameters are obtained after $3^{\text {rd }}$ level decomposition of input images as follows:

Table 1. Parameters of Input Images

\begin{tabular}{|c|c|c|}
\hline Parameters & Image 1 & Image 2 \\
\hline Entropy & 4.6623 & 5.9713 \\
\hline Mean & 71.1895 & 59.8007 \\
\hline Standard Deviation & 50.5549 & 56.2024 \\
\hline Variance & $2.5558 \mathrm{e}^{+03}$ & $3.1587 \mathrm{e}^{+03}$ \\
\hline
\end{tabular}

Table 2. Parameters of Fused Image

\begin{tabular}{|c|c|c|c|}
\hline Parameters & Haar & Daubechies & Symlet \\
\hline Entropy & 0.0028 & 0 & 0 \\
\hline Mean & 81.3428 & 81.6437 & 81.6350 \\
\hline Standard Deviation & 54.2340 & 54.3948 & 54.4575 \\
\hline Variance & $2.9416 \mathrm{e}^{+03}$ & $2.9588 \mathrm{e}^{+03}$ & $2.9656 \mathrm{e}^{+03}$ \\
\hline$M S E$ & $5.3644 \mathrm{e}^{-07}$ & $1.1296 \mathrm{e}^{-05}$ & $5.9630 \mathrm{e}^{-07}$ \\
\hline$P S N R$ & 62.7048 & 49.4706 & 62.2453 \\
\hline
\end{tabular}

\section{Conclusion}

In conclusion, using wavelet transform and weighted fusion, we get a good fusion image of CT/PET compared to the single CT or PET, we precisely discover and locate the diseased region in human body. It leads to accuracy which is far more improved and leads to extraction of maximum information as compared to other methods. Wavelet transform shows better resemblance with human visual system hence resulting in improved visualization and interpretation.

\section{Future Scope:}

Use of Computer Tomography (CT), Magnetic Resonance (MR), Single Photon Emission Computed Tomography (SPECT) techniques in biomedical imaging has revolutionized the process of medical diagnosis in recent years. Further advancements in biomedical imaging are being done by development of new tools of image processing. One of tools is image fusion. The fusion of CT scan, MR and SPECT images can make medical diagnosis much easier and accurate.

\section{References}

[1] C. Shangli, "Medical Image of PET/CT Weighted Fusion Based on Wavelet Transform", IEEE, (2009).

[2] R. Singh, M. Vatsa and A. Noore, "Multimodal Medical Image Fusion using Redundant Discrete Wavelet Transform", IEEE paper, (2009).

[3] Y. Yang, D. Sun Park, S. Huang, Z. Fang and Z. Wang, "Wavelet based Approach for Fusing Computed Tomography and Magnetic Resonance Images", CCDC, (2009).

[4] M. Sasikala and N. Kumaravel, "A comparative analysis of feature based image fusion methods", Information Technology Journal, vol. 6, no. 8, (2007), pp. 1224-1230.

[5] J. Daugman and C. Downing, "Gabor wavelets for statistical pattern recognition", The handbook of brain theory and neural networks, M. A. Arbib, ed. Cambridge, MA, USA: MIT Press, (1998), pp. 414-420.

[6] S. Mallat, "Wavelets for a vision", Proceedings of the IEEE, New York Univ., NY, vol. 84, no. 4, (1996) April, pp. 604-614. 
[7] A. Wang, H. Sun and Y. Guan, "The application of wavelet transform to multimodality medical image fusion", Proc. IEEE International Conference on Networking, Sensing and Control (ICNSC), Ft. Lauderdale, Florida, 2006, pp. 270-274.

[8] O. Rockinger, "Pixel-level fusion of image sequences using wavelet frames," Proc. of the 16th Leeds Applied Shape Research Workshop, Leeds University Press, (1996), 149-154.

\section{Authors}

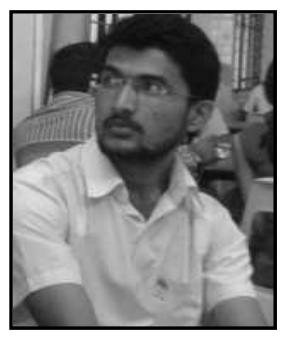

Laxman Tawade has pursued BE degree in Electronic \& Telecommunication from Pune University, India in 2011. Currently he is working as lecturer in VDF School of Engineering and Technology, Latur, India. He has done project internship at Reliance Innovation Communication Lab, Mumbai, India. He is member of International Association of Computer Science and Information Technology (IACSIT), Machine Intelligent Research Lab (MIR Lab), Universal Association of Computer and Electronics Engineers (UACEE), Academy and industry research collaboration center (AIRCC). He has 3 paper in international conference and 15 papers in International Journal to his credit. He has published papers in good reputed journals like Springer, Elsevier and John Wiley Son's Publication. He has worked as Reviewer for international journals and international conference sponsored by IEEE \& also worked as program committee member of few international conferences. He is working as technical program committee member of few international conferences. He is also invited as the Chair/Speaker of Network Security at the upcoming 2nd Annual World Congress of Emerging InfoTech 2013 (InfoTech-2013) at China. He is nominated for"Who's Who in the World". His research interest includes Image Processing ,Security \& Communication Network, Optical Fiber Communication and optical access networks based on WDM-PON, Biomedical signal processing and Power quality.

Abida Bapu Aboobacker is working as associate professor in Holy Grace Engineering College, kerala from 2012 till date. She was working with IES College of engineering from 2006 to 2012 as Assistant Professor cum head of department incharge. She had worked in Almihad Institution Alrigga, dubai from 2004 to 2005 as IT department in charge. She has worked as lecturer in Ansar Computer College from 2002 to 2004 for MCA students. She was incharge of R\&D Lab, AICTE, DTE, IEEE students chapter of the institution .She is member of IEEE and CSI. She also participated in various national level conferences .Her research interest include Image Processing, computer vision and Robotics.

Firdos Ghante BE passed from Bharat Ratna Indira Gandhi College of engineering in 2011. She is pursuing ME from Sinhgad academy of engineering, Pune, India. Her specialization is in Image Processing. 